\title{
Experimental study of gas injected bubble growth from submerged orifices
}

\author{
Sergio Di Bari, Anthony J. Robinson* \\ Department of Mechanical Engineering, Parsons Building, Trinity College, Dublin, Ireland
}

\section{A R T I C L E I N F O}

\section{Article history:}

Received 4 April 2011

Received in revised form 5 June 2012

Accepted 10 June 2012

Available online $\mathrm{xxxx}$

\section{Keywords:}

Bubble growth

Bubble departure

Submerged orifice

\begin{abstract}
A B S T R A C T
This work investigates the mechanism of adiabatic gas bubble growth from submerged orifices with the view of elucidating the interdependence of the bubble shape and the pressure field during growth and departure. Air injection flow rates between $10 \mathrm{mlph}$ and $100 \mathrm{mlph}$ have been tested for orifices of diameters $0.58 \mathrm{~mm}, 1.05 \mathrm{~mm}$ and $1.6 \mathrm{~mm}$. The force field around single isolated bubbles during growth and detachment has been investigated experimentally with the aid of high speed photography and detailed image processing and measurement of the internal bubble pressure. Different flow rates have been compared in order to elucidate dynamic effects. Combining measurements of the instantaneous gas pressure, measurements of the local curvature and the Young-Laplace equation has allowed the estimation of the liquid pressure field acting on the gas-liquid interface over the entire bubble surface. These have subsequently been decomposed into constitutive components such as buoyancy, contact pressure, capillary and the dynamic pressure forces.
\end{abstract}

(c) 2012 Elsevier Inc. All rights reserved.

\section{Introduction}

Nucleate boiling is a widely used mode of heat transfer. Even though boiling heat transfer is used in a vast range of domestic and industrial technologies, global theories and empirical formulations seem to be elusive. This is largely due to a lack of a complete understanding of the fundamental physics of bubble dynamics, flow and heat transfer at these small time and length scales. This is complicated by the fact that bubble dynamics are sensitive to a vast array of interdependent parameters which makes exhaustive experimentation and exact numerical simulations difficult to achieve.

Since the boiling phenomenon is so complex it is often difficult to isolate and understand the influence of the various parameters individually. With regard to bubble dynamics, an obvious approach is to inject gas bubbles into liquids with both fluids at the same temperature. This adiabatic approach has the advantages of being able to control the mass flux and the triple contact line as well as not having thermally induced convection (buoyant and/or thermocapillary) and other thermal aspects present. In this way the bubble dynamics can be scrutinised and the influence of well controlled parameters understood unambiguously. This being the case, there has been a great deal of experimental work done with regard to adiabatic gas injected bubble growth dynamics [1-16]. Some work has been performed on mathematically predicting adiabatic bubble formation and include the use of the boundary integral method [17-21], the Lattice-Boltzmann method [22] and numerical integration of the capillary equation [23-25]. More recently,

\footnotetext{
* Corresponding author. Tel.: +353 18963919; fax: +353 16795554 .

E-mail address: arobins@tcd.ie (A.J. Robinson).
}

CFD simulations have been performed to study various aspects of adiabatic bubble formation [26-33] on submerged orifices and nozzles and the results show that this is a very promising avenue for research in this branch of physics.

What is known from the body of work on bubble growth is that during bubble growth, the gas/vapour, liquid and solid phases interact dynamically at the bubble interface. Around the bubble dome the relative forces acting on the bubble interface determine the local curvature and aid in determining the bubble shape, motion and departure characteristics. At the triple contact line, the contact angle is dynamic and differs from the static contact angle which also plays a part in determining the bubble dynamics [34]. In this respect, mechanistic-based or numerical models which oversimplify the bubble geometry, such as assuming a constant apparent contact angle $[35,36]$ or assuming that the bubble remains a section of a sphere $[37,38]$ can, in many instances, not be representative of the actual physical phenomena since the Laplace pressure drop is sensitive to bubble shape [34].

There exists a fairly large body of work in the open literature which evaluate the forces acting on bubbles as they grow and depart from surfaces both during surface boiling [e.g. 39,40] and for gas bubbles growing from a needle or orifice [e.g. 15,17]. The various forces acting on bubbles, such as the surface tension, contact pressure, buoyancy, inertia and drag, can be evaluated from experimental data. This approach has been used to evaluate bubble growth and departure characteristics with varying degrees of complexity and success. Many of the forces mentioned, such as the buoyancy, surface tension and contact pressure forces are readily calculated from knowledge of the bubble shape. However, calculating the contribution of the dynamic pressure is not quite as trivial 


\section{Nomenclature}

\begin{tabular}{|c|c|}
\hline$a$ & Capillary Number (-) \\
\hline Bo & Bond Number (-) \\
\hline C & pressure constant $(\mathrm{Pa})$ \\
\hline$d$ & diameter $(\mathrm{m})$ \\
\hline$F$ & force $(\mathrm{N})$ \\
\hline$g$ & gravity vector $\left(\mathrm{m} / \mathrm{s}^{2}\right)$ \\
\hline$\stackrel{8}{h}_{b}$ & bubble height $(\mathrm{m})$ \\
\hline$H$ & liquid head $(\mathrm{m})$ \\
\hline$K$ & contact angle ratio $(-)$ \\
\hline$L$ & length scale $(-)$ \\
\hline$L_{c}$ & capillary length (m) \\
\hline$m$ & mass $(\mathrm{kg})$ \\
\hline$N_{p}$ & number of pixel $(-)$ \\
\hline$P$ & pressure $(\mathrm{Pa})$ \\
\hline$Q_{c r}$ & critical flow rate $\left(\mathrm{m}^{3} / \mathrm{s}\right)$ \\
\hline$R, r$ & radius $(\mathrm{m})$ \\
\hline$r^{\prime}$ & dimensionless radius $(-)$ \\
\hline$R_{1}, R_{2}$ & principal radii of curvature $(\mathrm{m})$ \\
\hline$t$ & time $(\mathrm{sec})$ \\
\hline$u$ & velocity $(\mathrm{m} / \mathrm{s})$ \\
\hline V & volume $\left(\mathrm{mm}^{3}\right)$ \\
\hline$V^{\prime}$ & dimensionless volume $(-)$ \\
\hline$V_{T}$ & Tate volume $\left(\mathrm{m}^{3}\right)$ \\
\hline$y$ & local vertical coordinate (m) \\
\hline$z$ & global vertical coordinate $m(-)$ \\
\hline
\end{tabular}

$\begin{array}{ll}\Delta P_{m} & \text { difference or pressure measured }(\mathrm{Pa}) \\ \text { Greek } & \\ \theta & \text { contact angle }\left(^{\circ}\right) \\ \rho & \text { density }\left(\mathrm{kg} / \mathrm{m}^{3}\right) \\ \sigma & \text { surface tension }(\mathrm{N} / \mathrm{m})\end{array}$

Suffixes

o orifice

atm atmosphere

$b \quad$ bubble

B buoyancy

C capillary

$C P \quad$ contact pressure

D dynamic

det detachment

g gas

gr bubble growth time

$l \quad$ liquid

$p \quad$ pixel

pr power law

$T \quad$ bubble tip or Tate

YP Young-Laplace

$s \quad$ static since it depends on a complex flow interacting with the bubble. As a first approximation added mass coefficients are often introduced for idealised geometries [41] or modifications of the extended Rayleigh equation for spherical bubble expansion are implemented to obtain an estimation of the dynamic pressure [42,43]. More recently, Chen and Groll [34] took advantage of the fact that sessile bubbles or pendent drops in a gravity field can be completely described by the capillary equation. In this work the bubble curvature, combined with an empirical correlation with adjustable parameters to best represent measured bubble profiles, were used to obtain information about the dynamic pressure field around the bubbles as they grew and departed from surfaces. It was determined that the dynamic pressure force played a very important role in determining the bubble shape and is thus intimately linked with the bubble dynamic behaviour.

This investigation carries forward from the analysis technique developed by Chen and Groll [34] to determine the pressure field around bubbles being emitted from a submerged orifice into a pool of water. This work will focus on quasi-static bubble growth and the influence that the air injection rate and orifice diameter have on the bubble growth and departure characteristics.

\section{Modelling of the forces and stresses acting on a growing bubble}

\subsection{Forces acting on a growing bubble}

A generic bubble can be viewed as a body sitting atop a horizontal surface, as depicted in Fig. 1. It is possible to divide the bubble surface into a dry area, coincident with the contact area immediately above the orifice, and a wet area which defines the gas-liquid interface. The bubble is fixed to the wall at the contact line defined by the rim of the orifice.

In the ensuing analysis of the bubble dynamics several simplifying assumptions have been made in order to quantify the forces acting on the bubble. These are listed as follows:
- All the fluid properties are considered constant and evaluated at room temperature.

- Bubble growth is occurring adiabatically and axissymmetrically.

- The rate of change of vertical momentum of the gas bubble is negligible compared with the magnitude of the vertical forces acting on it.

- The gas pressure is uniform within the bubble and the gas viscous and inertia influences are negligible.

- The contact line remains fixed to the rim of the orifice.

Since the injection rate and the gas density are very small the gas momentum is negligible compared with the static forces. This is confirmed by the calculation that the injection rate is below the critical injection rate outlined in [17], after which gas momentum is no longer negligible. As will be shown later, the assumption is confirmed by the small variation of the salient growth characteristics even with a 10 times increase in the gas injection rate.

Applying the above assumptions reduces the momentum balance on the bubble to what can be considered an equilibrium force balance which takes the form $[44,45]$;

$\sum \mathbf{F}=\mathbf{F}_{B}+\mathbf{F}_{C P}+\mathbf{F}_{C}+\mathbf{F}_{D}=0$

The principle forces acting on the bubble are the partial buoyancy force, $\mathbf{F}_{B}$, the contact pressure force, $\mathbf{F}_{C P}$, the capillary or surface tension force, $\mathbf{F}_{C}$, and the dynamic force, $\mathbf{F}_{D}$.

The bubble contact diameter is measured in this study. It was found that the bubble foot diameter remained fairly constant during the bubble lifetime. As a result, the upwardly directed net hydrostatic force only acts on the volume which exists in the region radially outward from the triple contact line, i.e. the shaded grey region in Fig. 1. This is to be termed the partial buoyancy force in order to avoid confusion with the classical definition posed by Archimedes. The partial buoyancy force is here defined as,

$F_{B}=\rho_{l} g V_{B}$ 

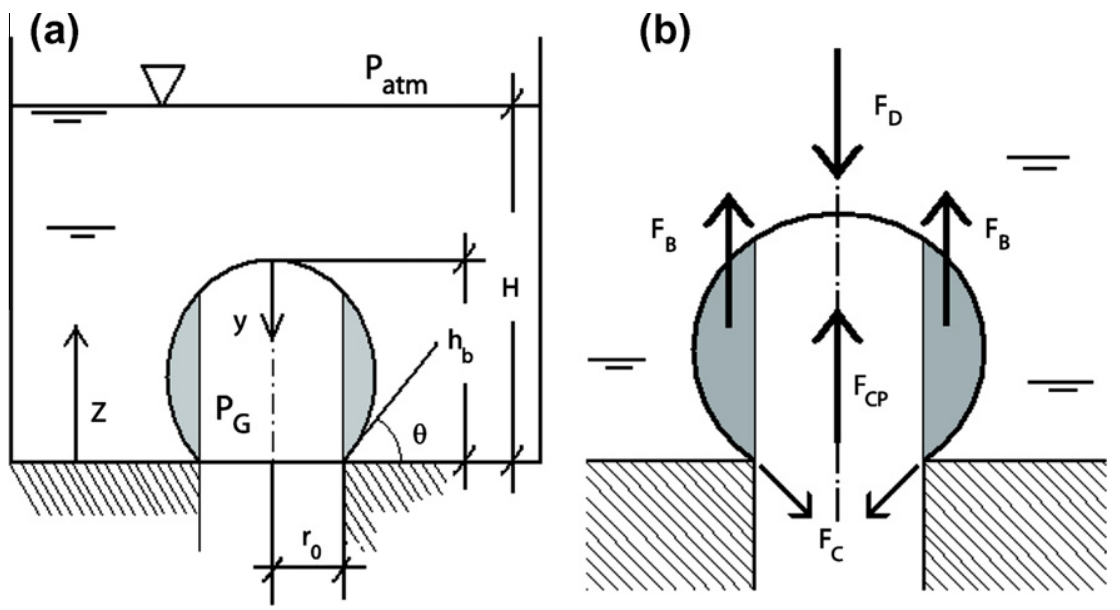

Fig. 1. (a) Bubble growing from a submerged orifice, and (b) forces acting on a growing bubble.

where $V_{B}$ is the volume section of the bubble with liquid both above and below it. For the case when a bubble neck has formed beneath the bubble, $V_{B}$ is determined from the section existing above the minimum neck radius.

The contact pressure force is due to the action of the overpressure inside the bubble acting on the projected area of the bubble tip above the orifice and is expressed as,

$F_{C P}=\pi r_{o}^{2}\left(P_{g}-P_{T}\right)$

where $P_{T}$ is the liquid pressure value at the bubble tip and $r_{0}$ is the contact radius. The choice of using $P_{T}$ in Eq. (3) simplifies the calculation of the integral of the liquid pressure acting on the upper region of the bubble.

The capillary force is a downwardly directed force that tends to keep the bubble attached to the wall. It arises due to capillary action at the triple interface and is related to the contact perimeter of the bubble and contact angle,
$F_{C}=2 \pi r_{0} \sigma \sin \theta$

where $\sigma$ is the liquid surface tension and $\theta$ is the contact angle at the triple contact line.

Finally, the dynamical influence of the moving liquid adjacent to the gas-liquid interface in the second term of the RHS of Eq. (1) are, for convenience, grouped into a single force term called the dynamic force and are a result of inertial and viscous effects,

$F_{D}=F_{\text {inertia }}+F_{\text {viscous }}$

Fig. 1b illustrates the forces schematically. Very detailed information concerning the flow is required to quantify the dynamic force. However, for cases for which the force balance of Eq. (1) is applicable, the dynamic force can be estimated provided that there is sufficient information available to calculate all of the other forces;

$F_{D}=F_{C}-\left(F_{C P}+F_{B}\right)$

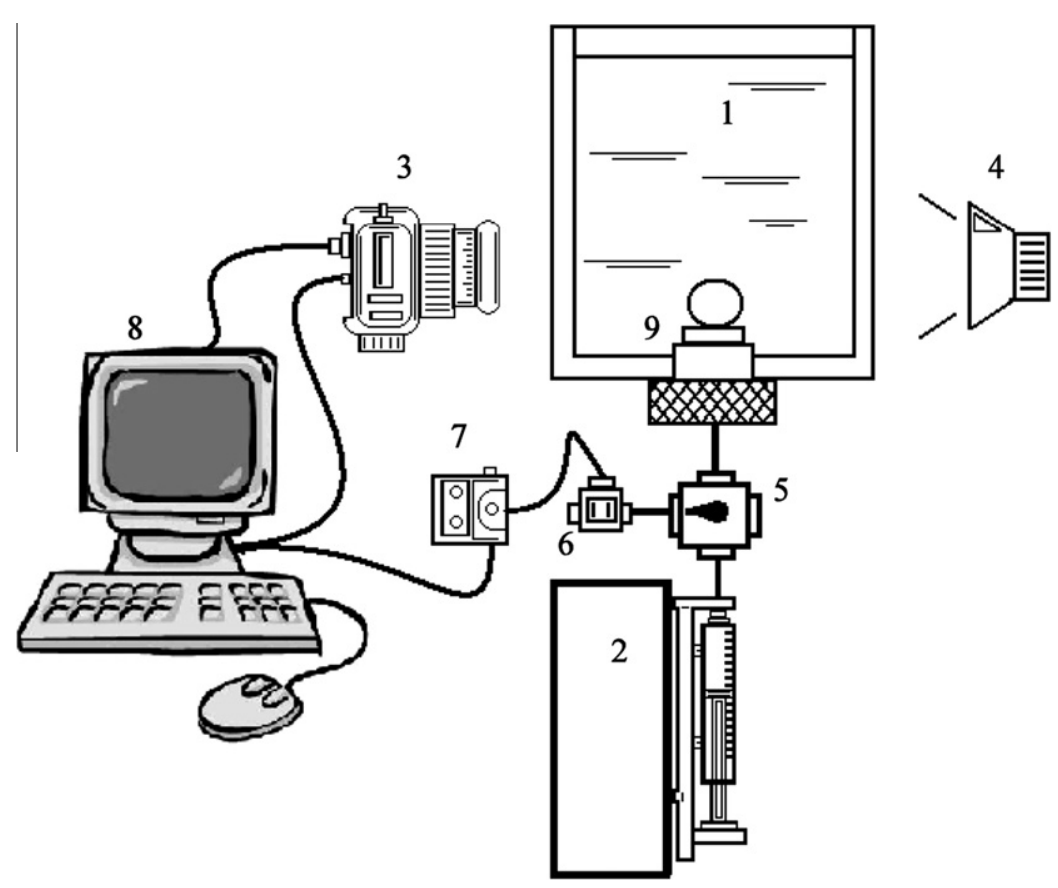

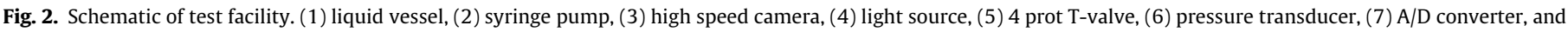
(8) data acquisition computer. 
(a)

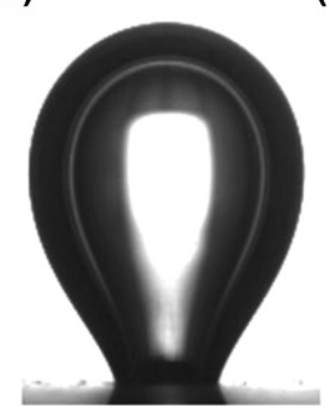

(b)

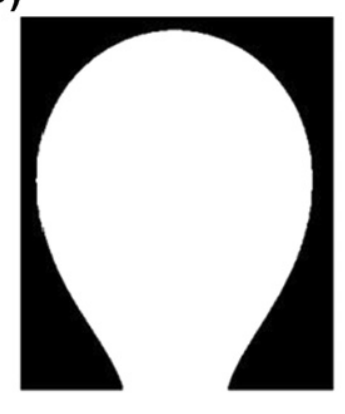

(c)

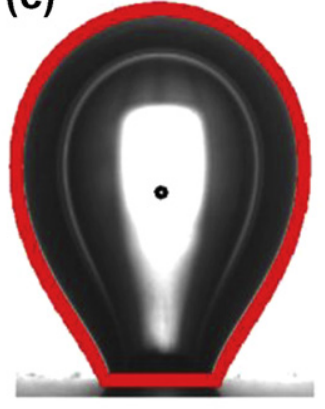

(d)

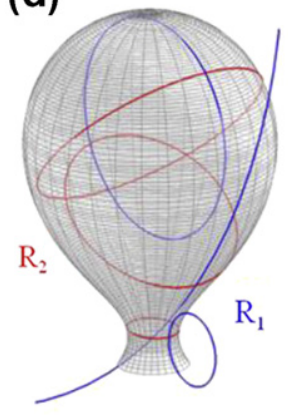

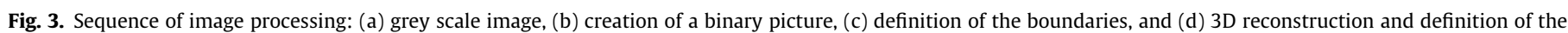
radii of curvature.

\subsection{Local stresses acting at the gas-liquid interface}

The magnitude of the pressure difference across the curved gasliquid interface is characterised by the Young-Laplace equation;

$\Delta P=\sigma\left(\frac{1}{R_{1}}+\frac{1}{R_{2}}\right)$

where $\Delta P$ is the local difference of pressure while $R_{1}$ and $R_{2}$ are the principal radii of curvature.

If a gravity field is applied the hydrostatic force acting on the bubble increases in the direction of the gravitational field causing the bubble shape to deviate from a perfect spherical section. The shape of a bubble in a gravitational field is governed by the capillary equation [46-48];

$\sigma\left(\frac{1}{R_{1}}+\frac{1}{R_{2}}\right)=\frac{2 \sigma}{R_{T}}-\left(\rho_{l}-\rho_{g}\right) g y$

where $R_{T}$ is the radius of curvature at the tip of the bubble, $\rho_{1}$ and $\rho_{g}$ are the density of the liquid and the gas respectively, $g$ is the acceleration due to gravity and $y$ is the vertical distance from the bubble apex.

In the case of dynamic growth it is necessary to take into consideration the fact that liquid inertia effects may, in some circumstances, play a role on the bubble growth and shape. Chen and Groll [34] developed a relationship which relates the bubble curvature to the static and dynamic components of the pressure,
$\sigma\left(\frac{1}{R_{1}}+\frac{1}{R_{2}}\right)=\frac{2 \sigma}{R_{T}}-\left(\rho_{l}-\rho_{g}\right) g y-\Delta P_{D}$

where $\Delta P_{D}$ is the local dynamic pressure difference including the contribution at the bubble tip,

$\Delta P_{D}=\left(P_{g D}-P_{l D}\right)-\Delta P_{T D}$

where $\left(P_{g D}-P_{I D}\right)$ is the local dynamic pressure difference while $\Delta P_{T D}$ is the dynamic pressure difference at the apex of the bubble.

\section{Experimental apparatus and image processing}

The experimental apparatus illustrated in Fig. 2 has been commissioned which can simultaneously measure the internal gas pressure and the shape of the bubble during growth and detachment.

The bubbles grew at the bottom of a small basin made of Perspex with base dimensions of $60 \mathrm{~mm} \times 60 \mathrm{~mm}$ and a height of $60 \mathrm{~mm}$. The basin is large enough that the influence of the side walls can be considered negligible. The bubbles grew and departed from small orifices of diameters $0.58 \mathrm{~mm}, 1.05 \mathrm{~mm}$ and $1.6 \mathrm{~mm}$ drilled through an aluminium cylinder of $2 \mathrm{~cm}$ diameter. The top of the vessel is open to the atmosphere and the vessel is partially filled with water at room temperature to a nominal height of $20 \mathrm{~mm}$.

A constant volumetric flow rate of room temperature air was varied between $10 \mathrm{mlph}$ and $100 \mathrm{mlph}$ by a controllable Kd Scientific Model 200 syringe pump. The syringe was connected to the

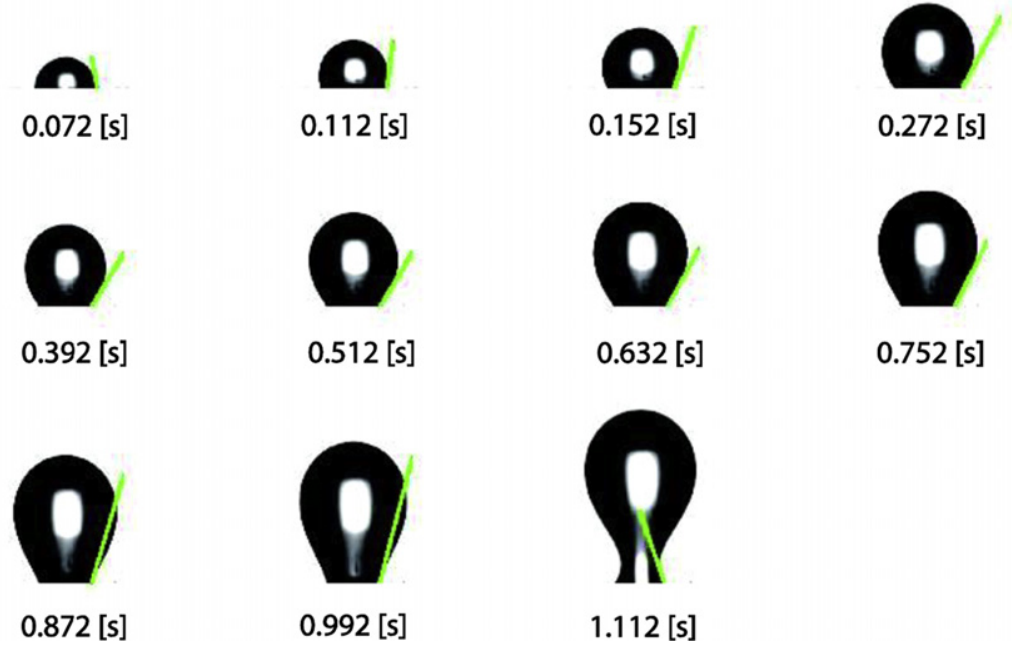

Fig. 4. Video sequence of bubble growth at $100 \mathrm{mlph}$ for $1.6 \mathrm{~mm}$ diameter orifice. 

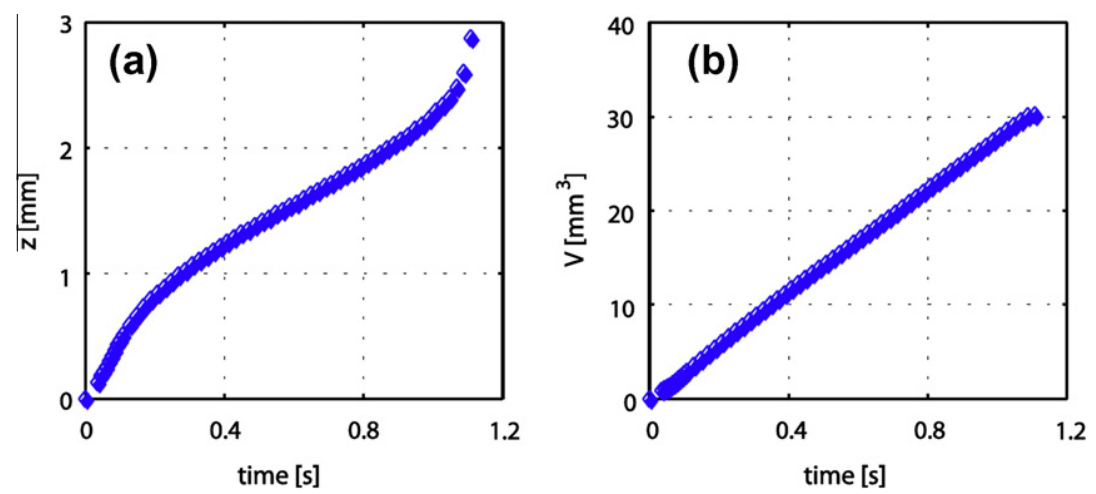

Fig. 5. (a) Motion of the centre of gravity and (b) volumetric growth at $100 \mathrm{mlph}$ for $1.6 \mathrm{~mm}$ diameter orifice.

orifice and to a pressure transducer through a "T" flow Path 4-ports Hamilton HV 4-3 so that the flow system did not need to be disconnected when drawing air back into the syringe.

In order to measure the pressure history inside the bubble, a fast response ( $\sim 1 \mathrm{~ms}$ ) Honeywell 164PC differential pressure sensor was joined to the $\mathrm{T}$ valve and measurements were acquired, processed and analysed with LabVIEW. The pressure transducer was calibrated against a U-tube manometer prior to testing.

All experiments were conducted under atmospheric condition and terrestrial gravity. Ultra pure deionised and distilled water was used which was boiled prior to testing to reduce dissolved gas content.

Having high quality images is fundamental to this work since the focus is not only on the principal geometric characteristics of the bubble, such as volume and equivalent area, but also on the bubble curvature and contact angle. Since the bubble growth is generally quite rapid, a NAC Hi-Dcam II high speed camera was utilised with frame rates ranging between $60 \mathrm{fps}$ and $1000 \mathrm{fps}$ depending on the bubble growth rate.

A purpose built Matlab code was written in order to define the relevant bubble characteristics such as the bubble volume, position of the centre of gravity, the coordinates of the bubble interface and the instantaneous contact angle. Fig. 3 illustrates the image processing sequence from the raw image to a 3D reconstructed bubble. The main image processing steps were the determination of a grey scale image (Fig. 3a), the choice of a threshold for the grey levels, the creation of a black and white picture using the threshold value (Fig. 3b) and then determining the bubble centroid and interface (Fig. 3c). As was discussed, knowledge of the two principal radii of curvature at each location on the bubble interface is required to quantify the pressure field acting upon it. This required further post-processing which involved the construction of a three dimensional bubble with a continuous surface which is illustrated in Fig. 3d. The 3D bubble was generated assuming axisymmetry and simply revolving the 2D image about the central vertical axis.

For every point on the bubble interface it is possible to approximate one of the principle radii of curvature by numerically fitting a circle that, by considering the neighbouring points, intersects that point such that the tangent to the circle is orthogonal to the outwardly directed normal vector of the interface. A simple routine was written in order to define the equation of the circumference passing through the generic point and its neighbouring points. Once the equation is known it is straight forward to find the value of the radius, and thus the curvature. The assumption of axisymmetry facilitates the formulation of the second radii of curvature which is equal to the length of the segment which extends from the interface to the symmetry axis in the direction normal to the interface.

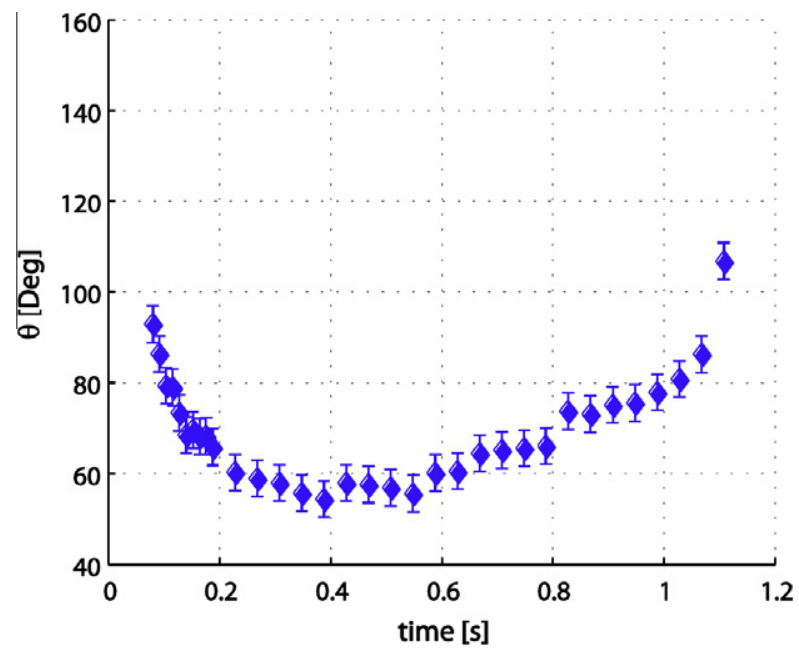

Fig. 6. Contact angle evolution at $100 \mathrm{mlph}$ for $1.6 \mathrm{~mm}$ diameter orifice.

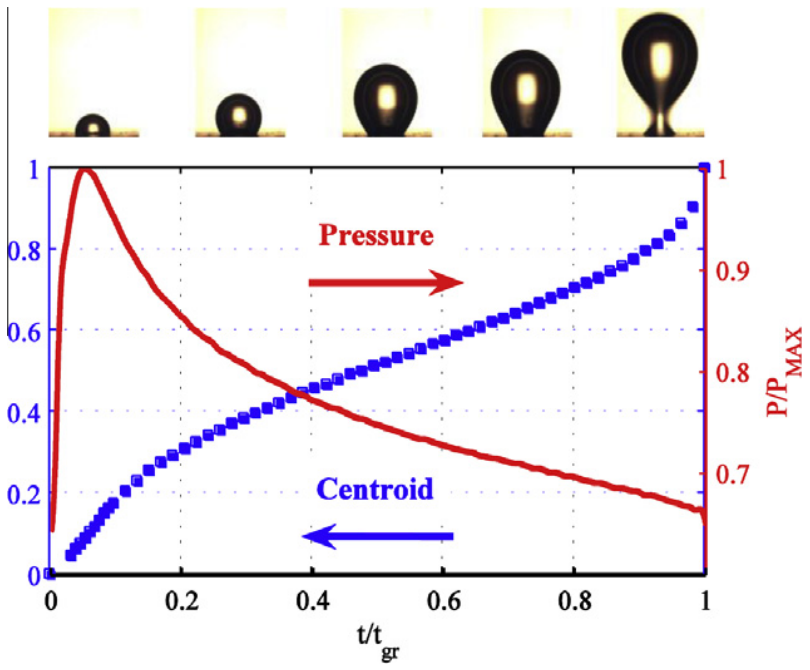

Fig. 7. Normalized centre of gravity position and differential pressure at $100 \mathrm{mlph}$ for $1.6 \mathrm{~mm}$ diameter orifice.

The gas pressure inside the bubble was measured and is given as,

$P_{g}=\Delta P_{m}+P_{a t m}$ 


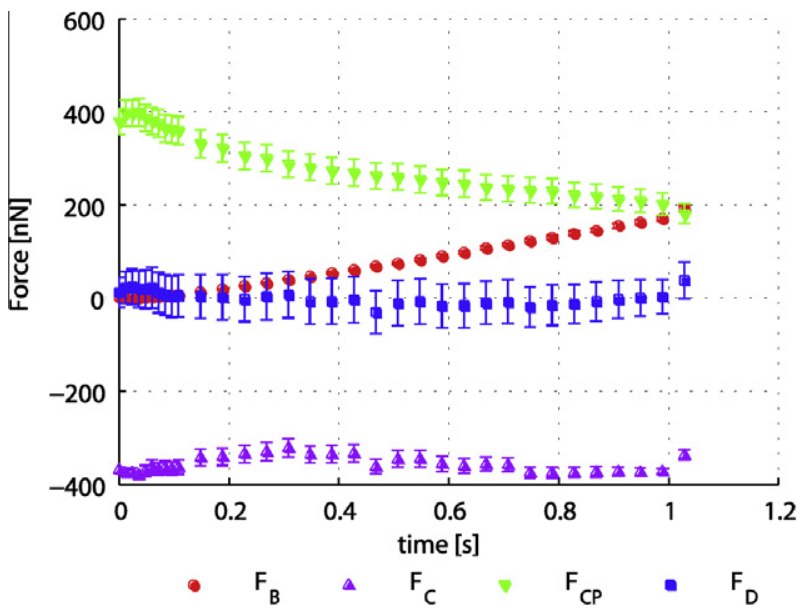

Fig. 8. Vertical forces acting on the bubble at $100 \mathrm{mlph}$ for $1.6 \mathrm{~mm}$ diameter orifice.

where $\Delta P_{m}$ is the measured differential pressure and $P_{a t m}$ is the atmospheric pressure. The local static pressure in the liquid at a vertical distance $y$ from the bubble tip is;

$P_{l s}=P_{a t m}+\rho_{l} g\left(H-h_{b}\right)+\rho_{l} g y$

where the first two terms account for the hydrostatic head between the free liquid surface and the apex of the bubble. Neglecting the gas inertia and substituting terms into the Young-Laplace equation, the local dynamic pressure can be estimated using the following relationship;

$P_{D}=\Delta P_{m}-P_{l}-\sigma\left(\frac{1}{R_{1}}+\frac{1}{R_{2}}\right)$

where $P_{l}=\rho_{l} \mathrm{~g}\left(H-h_{b}\right)+\rho_{l}$ gy represents the hydrostatic component
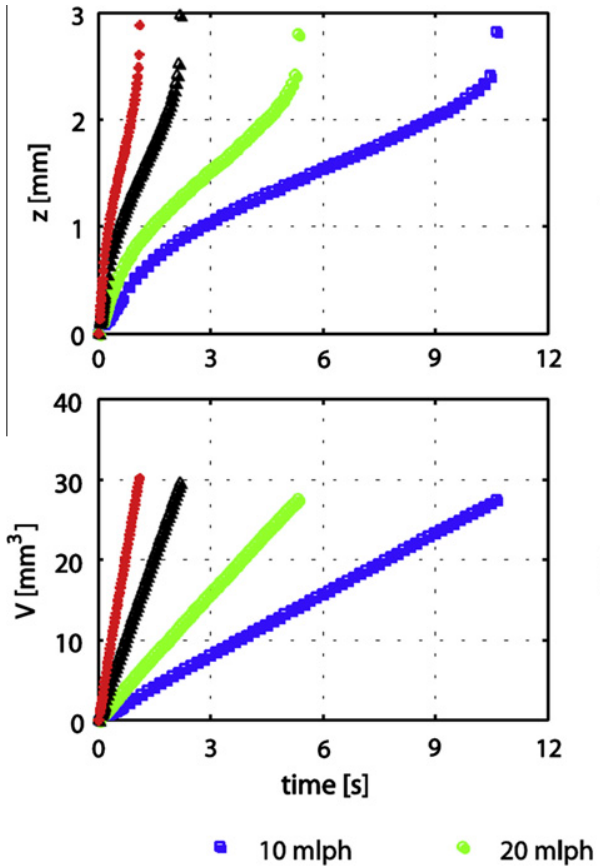
of the local pressure.

\section{Results and discussion}

\subsection{Bubble growth dynamics for the $1.6 \mathrm{~mm}$ diameter orifice}

Fig. 4 shows a photographic sequence of a bubble growing from the largest orifice tested for the highest air injection rate. The bubble evolution consists of three principal stages as is clear in Fig. 5a which is the time history of the centre of gravity. During the early stage, $(<0.2 \mathrm{~s})$, the bubble is emerging from the orifice and its shape is that of a spherical segment. Although the volumetric flow rate is constant, as confirmed in Fig. 5b, the bubble is decelerating. As the bubble volume increases a larger portion of the bubble is acted upon by buoyancy. When this portion of the bubble becomes large enough the bubble becomes progressively more elongated as the force tends to lift the upper portion of the bubble with the bubble foot remaining fixed to the orifice mouth. During this elongation/ expansion stage $(0.2 \mathrm{~s} \leqslant t \leqslant 0.8 \mathrm{~s})$ the rate of change of the centre of gravity is constant with time as shown in Fig. 5a.

For $t>0.8 \mathrm{~s}$ the bubble is in the process of detaching by forming a neck near the bubble base. Now, the influence of buoyancy is enhanced as the curving inward of the neck increases the volume being acted upon by buoyancy. This causes the upper region to accelerate upward as depicted in Fig. 5a where it is noticed that the centre of gravity accelerates upward prior to departure. The acceleration is caused by what is essentially a positive feedback effect whereby the collapsing of the neck of the bubble is responsible for increasing the rate of the necking process by increasing the magnitude of the partial buoyancy force. This eventually leads to break-off of the bubble from the collapsed neck.

Fig. 4 also depicts the instantaneous contact angle whose time evolution is plotted in Fig. 6. From the figure is it possible to clearly identify the three stages of the bubble growth defined by a different behaviour of the contact angle, which has been observed by other researchers $[15,49]$. As the bubble emerges from the orifice the contact angle decreases rapidly and changes from an obtuse angle to an acute angle. The partial buoyancy force increases in magnitude until the contact angle reaches a minimum and somewhat steady value of $60^{\circ}$ which is more or less maintained during
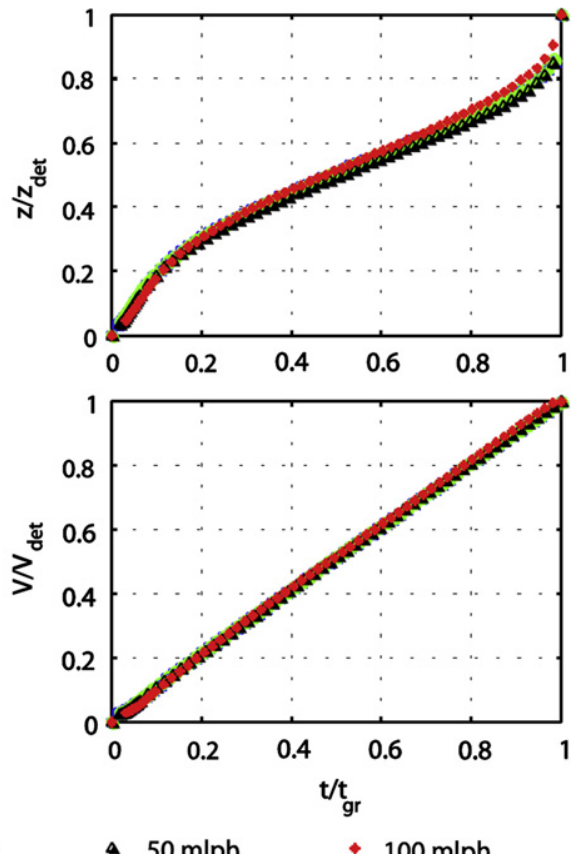

Fig. 9. Dimensional and non-dimensional motion of the centre of gravity (top) and volumetric growth rate (bottom). 
(a)

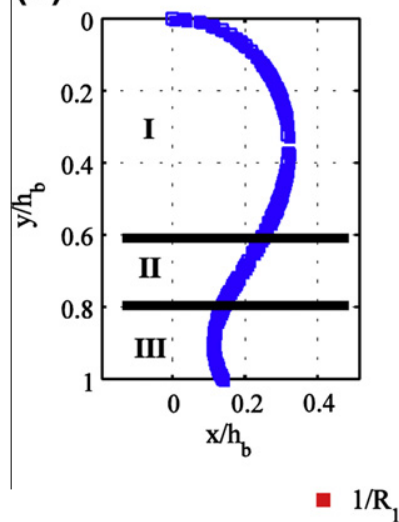

(b)

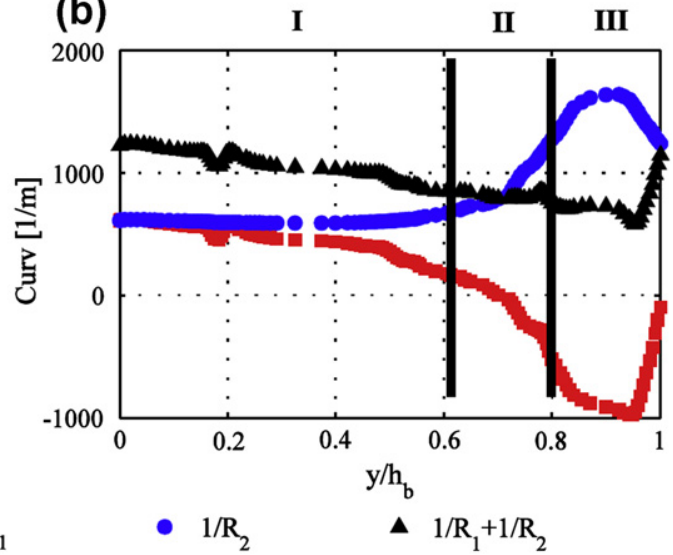

\section{(c)}

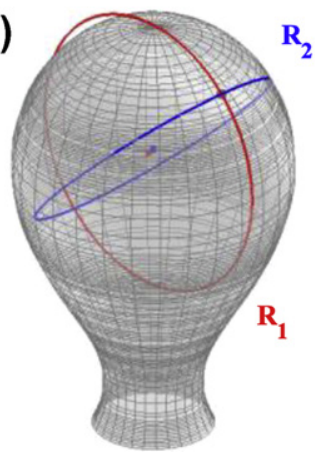

Fig. 10. (a) Vertical shape profile, (b) curvature characteristics and (c) 3D reconstruction of a bubble near departure.

the bubble elongation phase. This lasts until the partial buoyancy force acting on the upper portion of the bubble is so large that it pulls the bubble dome region upward with enough force to form a neck. As the neck forms the contact angle increases and once again becomes obtuse as it begins to pinch off, indicating bubble departure.

The differential pressure transducer facilitated the measurement of the gas pressure within the bubble while it is growing. The differential pressure history is given in Fig. 7. In agreement with the Young-Laplace equation, the maximum value of the pressure corresponds with the minimum radius which occurs when the bubble is hemispherical with its radius of curvature equal to the orifice radius. As the bubble expands and grows past the hemispherical shape the equivalent radius of the bubble increases and thus the pressure difference decreases monotonically to departure.

Using Eqs. (2), (3), (4) and (6) is it possible to approximate the vertical forces acting on the bubble as it grows. They are plotted with their relative uncertainty bars as a function of time in Fig. 8. The results are qualitatively similar to the force evolution trends of Duhar and Colin [15] for their quiescent bubble growth test case.

As discussed, the upwardly directed partial buoyancy force, $F_{B}$, is equal to zero for approximately $t<0.1 \mathrm{~s}$ as there is no liquid underneath the bubble until the contact angle drops below $90^{\circ}$ and the bubble resembles a truncated sphere. From this point onward the upward partial buoyant force acts on the region of the bubble that exists radially outward from the triple contact line and increases at a nearly constant rate and is a significant force during the bubble elongation and departure phases.

The upwardly directed contact pressure force, $F_{C P}$, due to its dependency on the differential pressure, is maximum near the beginning of the bubble growth when it is hemispherical and the pressure difference is maximum (Fig. 7). Subsequent to this point in time this force monotonically decreases, generally following the trend of the gas pressure evolution.
The capillary force, $F_{C}$, is downwardly directed and acts to keep the bubble fixed to the wall. It has an absolute maximum value when the bubble is hemispherical in shape as the sine function in Eq. (5) is unity. Its magnitude is not, however, characterised by major changes during the different growth stages and tends to remain relatively constant.

The dynamic force, $F_{D}$, is calculated from the resulting force balance (Eq. (6)). Due to error propagation the uncertainty associated with the dynamic force is largest compared with the others. However, Fig. 8 confirms the quasi-static nature of the bubble growth as the dynamic force is, within the context of the experimental uncertainty, negligibly small during the entire bubble life.

Fig. 9 shows the bubble centre of gravity and volume curves for varying gas injection rates for both dimensional (left) and nondimensional (right) scales. For the cases considered here the gas injection rates between $10 \mathrm{mlph}$ and $100 \mathrm{mlph}$ are below the critical value defined in [17] as,

$Q_{c r}=\pi\left(\frac{16}{3 g^{2}}\right)^{1 / 6} \cdot\left(\frac{\sigma \cdot r_{0}}{\rho}\right)^{5 / 6}$

beyond which the flow rate is known to influence the growth and departure characteristics. This being the case, the bubble detachment volume does not depend on the gas injection rate with an average departure volume of $V_{\text {det }}=29 \mathrm{~mm}^{3} \pm 3 \%$. Likewise, there is no noteworthy influence of the flow rate on the vertical forces histories depicted in Fig. 8.

Fig. 9 shows that the non-dimensional centre of gravity curves show very good collapse indicating that for the quasi-static growth, the bubble shape depends on the instantaneous bubble volume only. Similar behaviour with regards to the departure diameter and bubble centre of gravity has also been observed in [50] for their low Jakob number bubble growth experiments from an artificial nucleation site during boiling where the volumetric growth rate was not constant with time. 

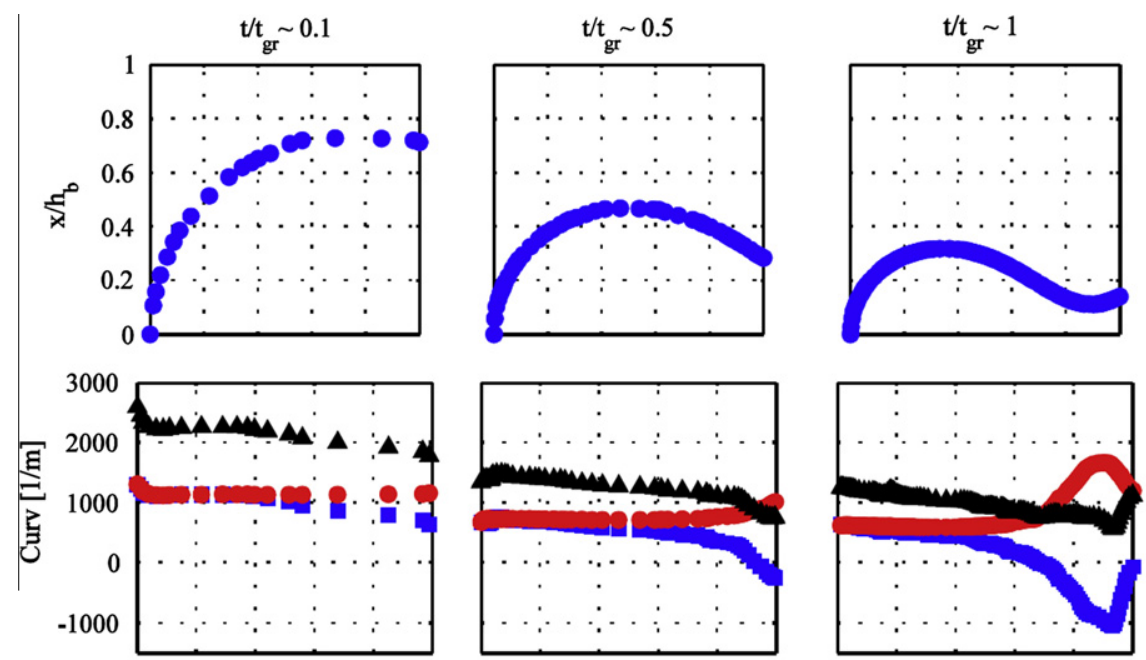

- $1 / \mathrm{R}_{1}$

- $1 / \mathrm{R}_{2}$

- $1 / R_{1}+1 / R_{2}$
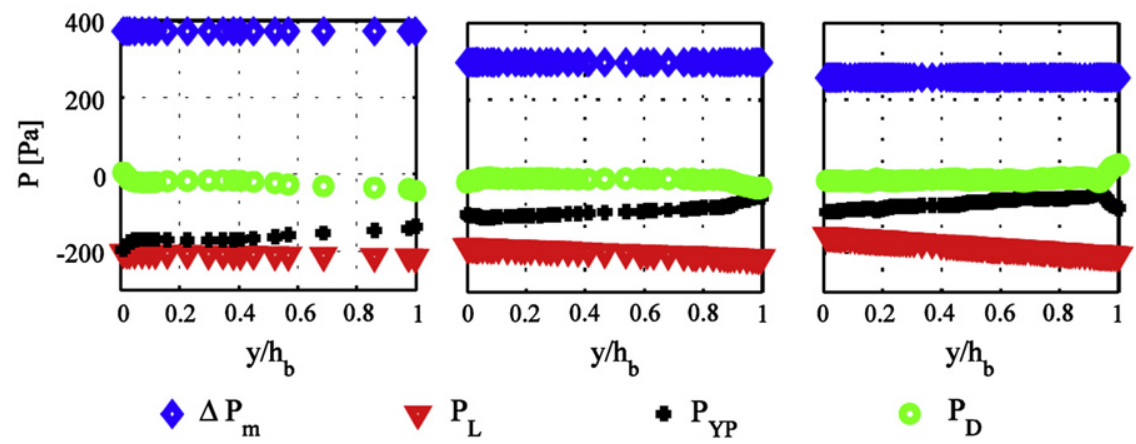

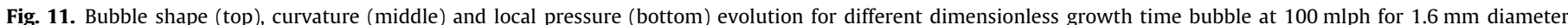
orifice.

Fig. 10 illustrates the distribution of the inverse of the principle radii of curvature, $1 / R_{1}$ and $1 / R_{2}$, with dimensionless distance $y / h_{b}$, where the vertical coordinate, $y$, has its origin at the apex of the bubble and increases positively in the direction of gravity, and $h_{b}$ is the instantaneous bubble height. A positive curvature corresponds with the situation whereby the centre of the circle which defines the principle radius is situated to the gas side of the interface. Further to this, it is worth clarifying that $R_{1}$ is the local radius with respect to a vertical plane cutting the bubble along its vertical axis, whilst $R_{2}$ lies on the orthogonal plane to that of $R_{1}$ (Fig. $3 \mathrm{~d}$ and 10c).

Depending on the growth stage, it is possible to divide the bubble into three distinct sections. This is depicted in Fig. 10 for a bubble near departure where the neck has formed near the bubble base. Region I, which for this instance is approximately in the range $0 \leqslant y / h_{b} \leqslant 0.6$, is the largest section of the bubble. It has an elongated spherical shape in which $R_{2}$ remains approximately constant. The magnitude of $R_{1}$ is close to that of $R_{2}$ so it can be said that Region I is defined by a nearly spherical segment. Even still, $R_{1}$ tends to increase slightly in this region causing the curvature to decrease as well. The net effect is that Region I exhibits a moderate decrease in the overall curvature which is of course related to a decrease in the Laplace pressure drop across the interface.

The term $1 / R_{1}$ drops to zero as the shape changes from convex to concave in this plane and defines the beginning of Region II. Region II can be considered a transition region between the upper spherical dome (Region I) and the bubble neck (Region III). It is characterised by concaveness with respect to $R_{1}$ and a notable drop in $R_{2}$ as the bubble begins to pinch-off near the neck. Since the two curvatures act in opposite directions they have opposing influences on the Laplace pressure drop which tends decrease only slightly.

Region III only appears during bubble departure where the neck has formed and bubble pinch-off is occurring, i.e. when the centre of gravity is accelerating. It is important to mention that due to the size and speed of the necking phenomena the image processing quality was poorer in this lower region compared with the other two regions. Nonetheless, in Region III the evolutions of $1 / R_{1}$ and $1 / R_{2}$ achieve local minimum and maximum values respectively. In the top part of this region the Laplace pressure drop remains somewhat constant due to the offsetting effects of the two principle radii of curvature though is subject to a visible increase in the proximity of the bubble base.

The evolution of the curvature and the effects on the pressure field on the bubble interfaces are plotted in Fig. 11 for dimensionless times corresponding with $t / t_{g r} \sim 0.1,0.5$ and 1 . These are chosen to be indicative of the different stages of bubble growth. The sign convention in Fig. 11 is that positive values correspond with pressure directed outwardly on the bubble (i.e. gas pressure) while negative values correspond with pressure directed inwardly (i.e. liquid pressure).

For $t / t_{g r} \sim 0.1$, Fig. 11 indicates that the bubble is quite spherical. The term $1 / R_{1}$ tends to decrease slightly whilst $1 / R_{2}$ remains essentially constant such that the sum of the two, and thus the Laplace pressure is subject to a small decrease.

For a mid-growth bubble, i.e. the elongated sphere with a positive contact angle, Fig. 11 shows that the bubble remains essentially spherical in the region of the bubble apex. The subsequent drop in the Laplace pressure approaching the bubble base is due to a decrease in $1 / R_{1}$ though the drop is moderated somewhat by 


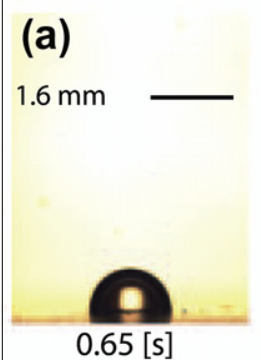

(b)

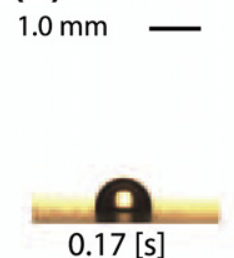

(c)

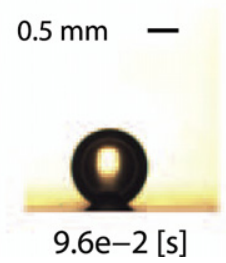

$2.26[s]$
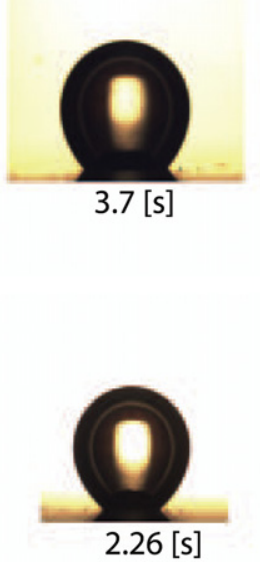

$3.7[s]$

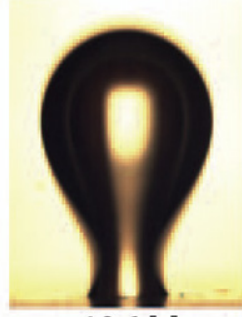

$10.6[s]$

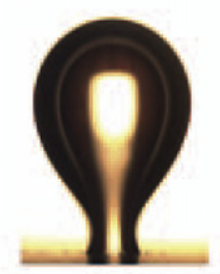

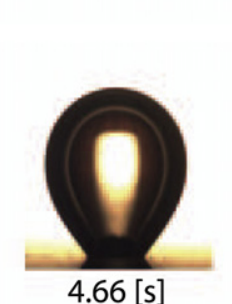

$4.66[s]$

$6.99[\mathrm{~s}]$

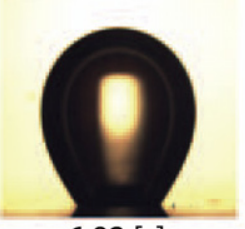

$6.92[s]$

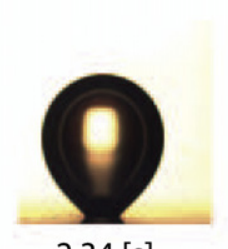

$2.34[s]$

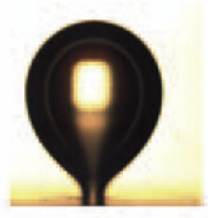

$3.55[s]$

Fig. 12. Photographic sequence of bubble formation for a gas injection rate of $10 \mathrm{mlph}$ and orifice diameter of (a) $1.6 \mathrm{~mm}(\mathrm{~b}) 1.05 \mathrm{~mm}$ and (c) $0.58 \mathrm{~mm}$.

a gradual increase in $1 / R_{2}$. For the late stage the curvature profiles depicted in Fig. 11 have already been discussed above.

The quasi-static nature of growth is evident from the negligible value of the dynamic pressure acting on the bubble interface for all the three non-dimensional times analysed. The linear increase of the Young-Laplace drop is in fact balanced by the linear decrease of the liquid pressure. An exception is noted in the proximity of the neck where the dynamic pressure is subject to a sizeable increase due to the sharp constriction of the neck. During a very short interval the concave radius $R_{2}$ decreases whereas the convex radius $R_{1}$ increases having a net effect of increasing the Laplace pressure. As the neck quickly collapses the surrounding liquid is set into motion to such an extent that hydrodynamic forces, i.e. viscous and/or inertial, become non-negligible.

\subsection{Effect of orifice diameter}

Orifice diameters of $0.58 \mathrm{~mm}, 1.05 \mathrm{~mm}$ and $1.6 \mathrm{~mm}$ were tested for the same range of gas injection rates. As evident in the photographic sequences shown in Fig. 12, the size of the orifice has a notable influence on bubble growth and departure characteristics. In general it is observed that the bubbles tend to be smaller, depart sooner and remain more spherical for the smallest orifice. For the larger orifices the bubbles tend to elongate vertically due to the action of buoyancy, as will be discussed, and depart with larger volumes and departure times.

The evolution of the bubble profiles and their curvatures are plotted respectively in Fig. 13 for the largest and the smallest orifices at the lowest flow rate. Again, non-dimensional times of $t /$ $t_{g r} \sim 0.1,0.5$ and 1 have been chosen to be indicative of the early, mid and late growth stages.

At $t / t_{g r} \sim 0.1$ the influence of the orifice diameter is already clear as the two bubble shapes are considerably different. The bubble from the largest orifice has a profile completely inside Region I due to its clear hemispheric shape $\left(1 / R_{1} \sim 1 / R_{2}\right)$ and, from Fig. 14 , the contact angle is close to $90^{\circ}$. A truncated spherical shape is evident for the bubble growing from the smallest orifice, with a contact angle of approximately $40^{\circ}$.

For mid-growth bubbles $\left(t / t_{g r} \sim 0.5\right)$ the two shapes resemble truncated spherical-type sections with some elongation due to the increasing influence of buoyancy. Region II begins to form at approximately $y / h_{b}>0.4$ as $1 / R_{1}$ decreases notably for both bubbles. Approaching the bubble foot $\left(y / h_{b}>0.8\right)$ the two bubble shapes begin to deviate from one another as evident from Fig. 13a. Here, the curvature $1 / R_{2}$ increases notably for the $0.58 \mathrm{~mm}$ orifice as $R_{2}$ approaches $r_{o}$ and indicates the early formation of a neck. As evident in Fig. 14, the contact angle has increased moderately from $40^{\circ}$ and continues to do so until the departure phase. The net effect on the overall curvature is a moderate drop over the upper bubble dome and a notable increase near the bubble foot where the neck is forming. Conversely, for the $1.6 \mathrm{~mm}$ orifice, the contact angle has dropped to around $60^{\circ}$ and the curvature, $1 / R_{2}$, increases only slightly near the bubble foot. Here, the influence of buoyancy is stronger since it acts on a larger volume causing the bubble to elongate and deviate further from a truncated spherical segment. The overall curvature decreases monotonically from the bubble tip to base.

For the late stage bubbles $\left(t / t_{g r} \sim 1\right)$ the curvature profiles for the $1.6 \mathrm{~mm}$ orifice have been discussed earlier. In comparison, there is a notable influence of the orifice size on the bubble neck profiles. For the larger diameter orifice the neck has associated with it an hourglass type of profile. In this region $1 / R_{1}$ decreases, becomes negative and reaches a local minimum where the width of the neck is thinnest, i.e. where $1 / R_{2}$ is maximum. Here the neck is collapsing which eventually ends in the complete pinching of the neck and bubble break-off. Beyond this inflection point $1 / R_{2}$ 

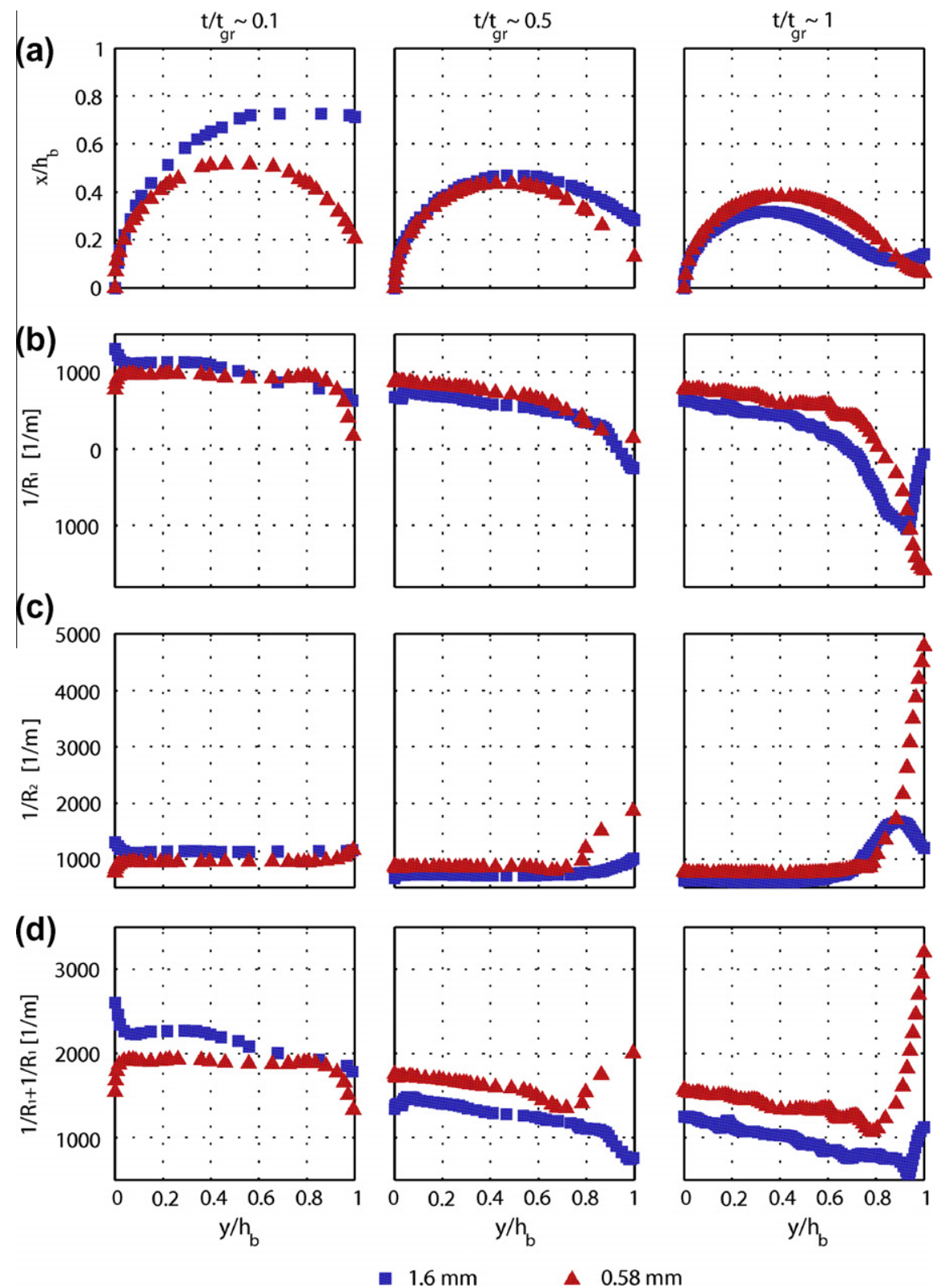

Fig. 13. Evolution of (a) bubble contours, (b) $1 / R_{1}$, (c) $1 / R_{2}$ and (d) $1 / R_{1}+1 / R_{2}$.

increases until $R_{2} \sim r_{o}$ causing $1 / R_{1}$ to increase and $1 / R_{2}$ to decrease respectively. Here, the contact angle depicted in Fig. 14 increases to become an obtuse angle as the neck collapses. Conversely, for the $0.58 \mathrm{~mm}$ orifice both $1 / R_{1}$ and $1 / R_{2}$ are approximately equal and uniform over the bubble dome indicating that it is nearly spherical. However, beyond $y / h_{b} \sim 0.8,1 / R_{1}$ decreases and $\mathrm{I} / R_{2}$ increases sharply. This is indicative of the short and nearly cylindrical neck which is evident in Figs. 12 and 13a, which is strikingly different than the neck profile of the largest orifice. As shown in Fig. 14 the contact angle increases but remains acute until the neck begins to collapse and the pinching and bubble break off event occurs. This occurs extremely fast for the small orifice compared with the largest orifice which is consistent with previous observations [51]. Overall, the curvature profiles, $1 / R_{1}+1 / R_{2}$, both tend to decrease moderately in magnitude moving downward from the tip with an increase near the bubble foot which is much more pronounced for the smaller orifice.
Fig. 15 illustrates the local pressures (Eq. (13)) acting along the bubbles corresponding with Fig. 13. Of course the Young-Laplace pressure $\left(P_{Y P}\right)$ profiles are related to the overall curvature profiles in Fig. 13. The static liquid pressures and the measured pressure differentials tend to be of similar magnitude. The dynamic pressure components are negligible for early and mid growth though, as discussed earlier, increase in the neck region near departure due to hydrodynamic forces as the neck collapses. Since the neck collapse event occurs much faster for the smaller orifice bubble, the magnitude of the dynamic pressure is notably larger than that of the larger orifice bubble.

Fig. 16 illustrates the evolution of the vertical forces for the case of the lowest flow rate. Apart from the dynamic forces which are negligible, the magnitude of the forces decrease with decreasing orifice diameter as do their relative magnitudes during the respective growth histories.

Considering Eq. (3), the magnitude of the contact pressure force decreases with orifice size since the pressure difference $\Delta P \propto 1 / r_{o}$ 


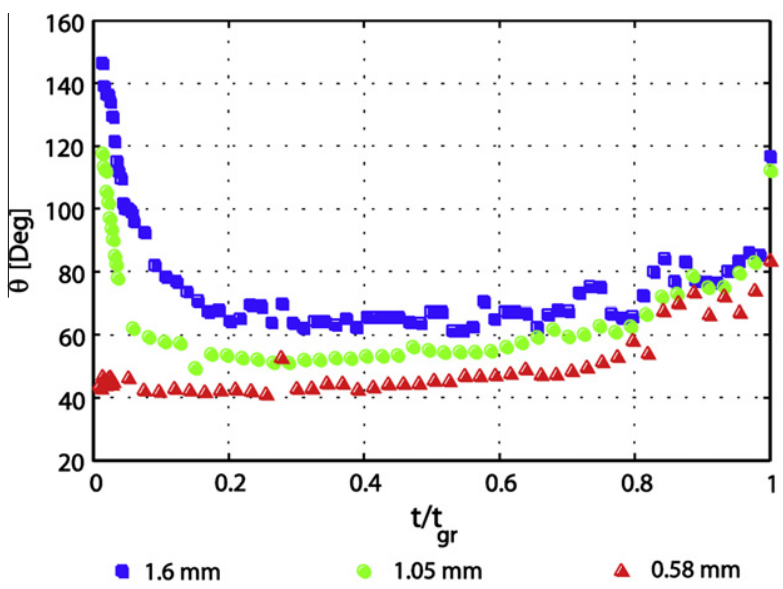

Fig. 14. Contact angle histories for varying orifice diameter at $10 \mathrm{mlph}$.

while the contact area $\mathrm{CA} \propto r_{o}^{2}$ thus the contact pressure force $F_{C P} \propto r_{o}$.

The magnitude of the capillary force (Eq. (4)) decreases with orifice diameter since the smaller contact perimeter is augmented by a smaller value of the contact angle as depicted in Fig. 14. The decreased contact angle is largely due to a decrease in the magnitude the partial buoyancy force with orifice size. The partial buoyancy force decreases simply due to the fact that the smaller orifice produces smaller bubbles (Fig. 12) with a corresponding decrease in the $V_{B}$ in Eq. (2). Unlike the larger orifices, the partial buoyancy force acts on the bubble emitted from the smallest orifice straight away since $\theta<90^{\circ}$ almost immediately. However, due to its smaller size it does not have as strong an influence on the bubble shape during early and mid growth.

An uncomplicated measure of the level of influence on the bubble shape is to consider the ratio, $K$, of the measured contact angle and the angle which would exist if the bubble were a perfect spherical segment of the same volume and bubble foot radius,

$K=\theta /(90-\sin (s / R))$ where $s$ and $R$ are the centre of curvature and radius of a perfect spherical segment respectively. For mid growth $\left(t_{g r} \sim 0.5\right)$ $K=1.25,1.55$ and 1.75 for the $0.58 \mathrm{~mm}, 1.05 \mathrm{~mm}$ and $1.6 \mathrm{~mm}$ diameter orifices respectively, indicating increased bubble deformation with the increased strength of buoyancy for increasing orifice size.

It is also clear from Fig. 16 that the relative magnitudes of the forces are largely influenced by the orifice size. In particular, the relative contribution of the contact pressure force diminishes with orifice diameter. For the largest orifice, the contact pressure is the dominant upwardly directed force for a large portion of the bubble lifetime and is approximately equal to the partial buoyancy force at departure. On the other hand, the contact pressure decreases below the partial buoyancy force for the smaller two orifices and the crossover point occurs earlier with decreasing orifice size. At departure it is evident that the contact pressure becomes progressively less influential with decreasing orifice size to the extent that, for the smallest orifice, it plays a minor role at departure. Since each liquid has a unique capillary length defined here as,

$L_{c}=\sqrt{\frac{\sigma}{\left(\rho_{l}-\rho_{g}\right) g}}$

it is practical to say that that the relative importance of the contact pressure force diminishes as the dimensionless ratio, $d_{o} / L_{c}$, decreases, in particular at departure since the bubbles are generally much larger than the orifice for small $d_{o} / L_{c}$.

This being the case, it is likely that the departure volume will approach the Tate Volume [52], $V_{T}$, for sufficiently small orifice diameters since it is derived from a balance of buoyancy and capillary forces with a departure contact angle in the region of $90^{\circ}$ such that;

$V_{T}=\frac{\pi d_{o} \sigma}{\left(\rho_{l}-\rho_{g}\right) g}$

Fig. 17 shows the measured departure volume versus orifice diameter for this work as well as those of Di Marco et al. [46] for air bubbles injected into water from orifices of $0.14 \mathrm{~mm}$ and $0.32 \mathrm{~mm}$ diameters. The Tate Volume is also plotted in the figure.
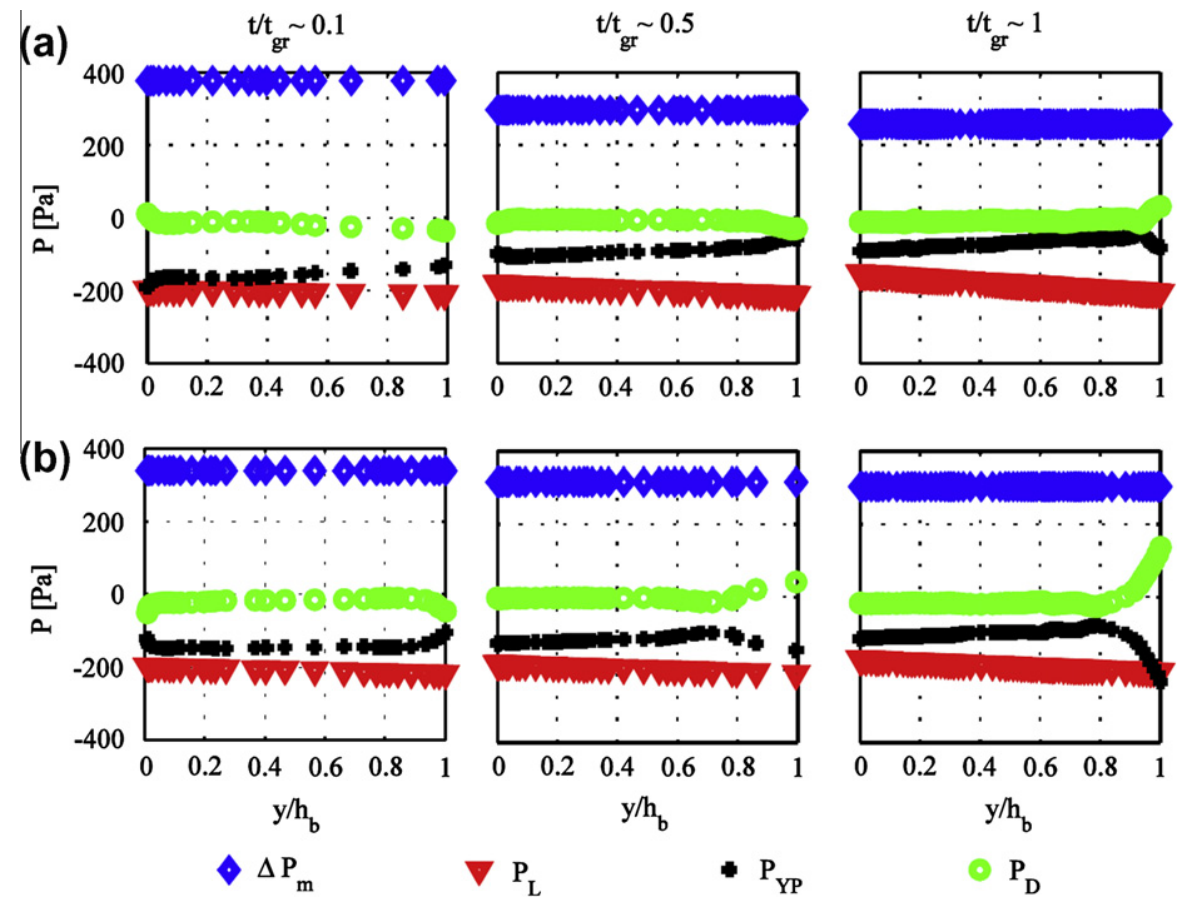

Fig. 15. Local pressures acting on the bubble interface for (a) $1.6 \mathrm{~mm}$ (b) $0.58 \mathrm{~mm}$ orifice diameters. 

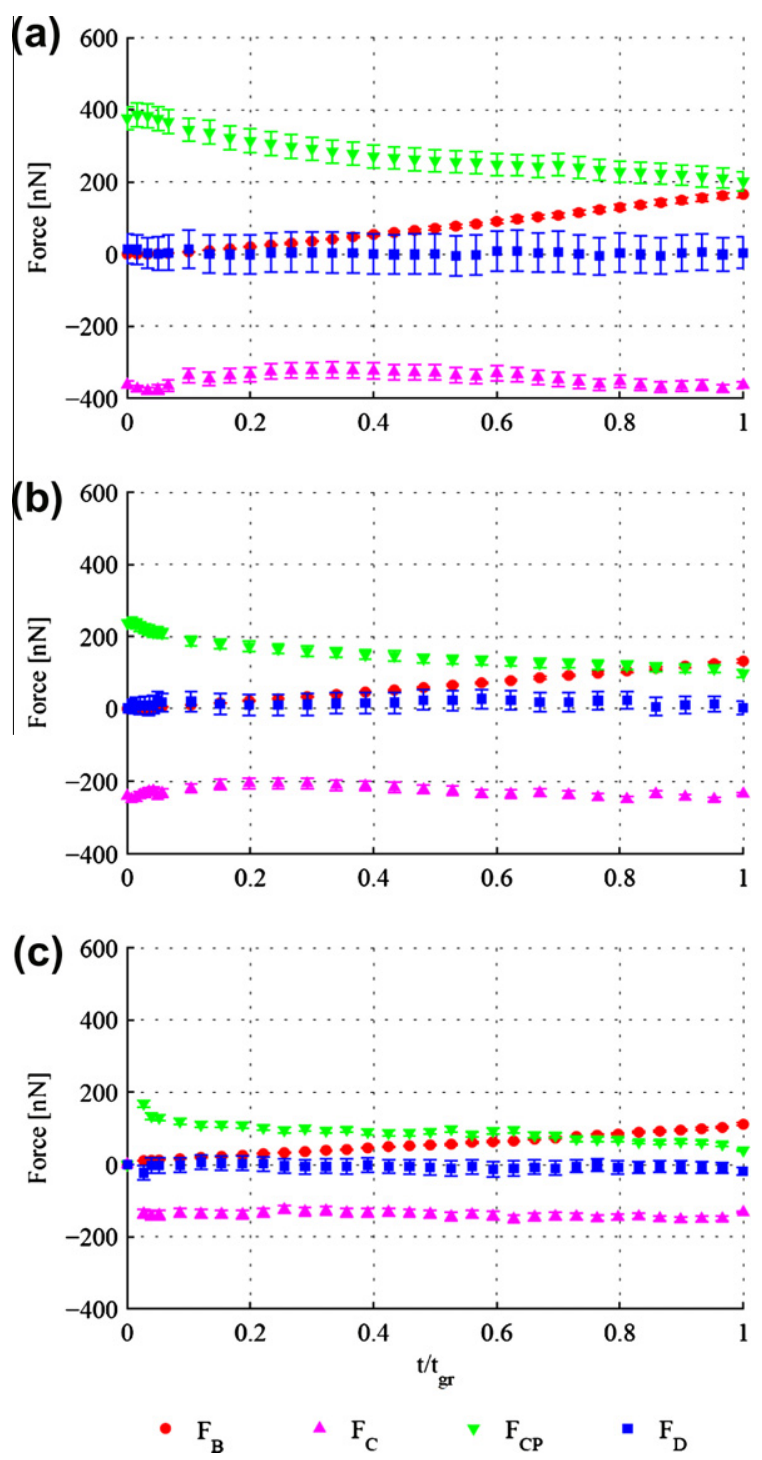

Fig. 16. Vertical forces acting on the bubble at $10 \mathrm{mlph}$ (a) $1.6 \mathrm{~mm}, d_{o} / L_{c}=0.58$ (b) $1.05 \mathrm{~mm}, d_{o} / L_{c}=0.31$ and (c) $0.58 \mathrm{~mm}, d_{o} / L_{c}=0.31$.

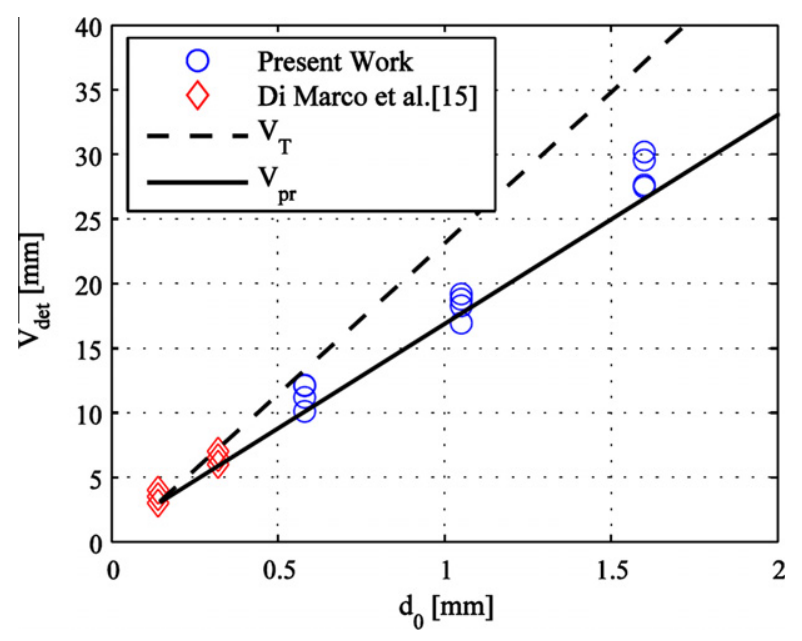

Fig. 17. Bubble departure volume versus orifice diameter.

It is clear that $V_{\text {det }} \rightarrow V_{T}$ for the smaller orifices. However, $V_{\text {det }}$ become progressively smaller than $V_{T}$ with increasing orifice diameter due to the increasing influence of the contact pressure. Since the relative importance of the contact pressure is related to $d_{o} / L_{c}$, a simple power law dependence to account its influence is given here as,

$V_{p r}=0.6863\left(\frac{d_{o}}{L_{c}}\right)^{-0.116} V_{T}$

where the data from this work for each orifice radius and all injection rates has been used in conjunction with the Di Marco [47] water data for the regression analysis. Fig. 17 shows good agreement for the water data for orifice diameters in the range of $0.14 \mathrm{~mm} \leqslant d_{o} \leqslant 1.6 \mathrm{~mm}$.

Non-dimensionalizing the departure volume with the orifice radius such that $V^{\prime}=V_{\text {det }} / r_{o}^{3}$ and $r^{\prime}=r_{o} / L_{c}$, and $B o=r_{o}^{2} g\left(\rho_{l}-\rho_{g}\right) / \sigma$ Eq. (18) can be rewritten in dimensionless form as,

$V_{p r}^{\prime}=3.78\left(r^{/}\right)^{-0.116} B^{-1}$

Fig. 18 shows very good agreement between the predictions of Eq. (19) and a broad range of data from different sources including several different fluids (water, ethanol, FC72, HFE7000), orifice diameters $\left(0.14 \mathrm{~mm} \leqslant d_{o} \leqslant 4 \mathrm{~mm}\right)$ and gravity levels $(0.5-1.5 \mathrm{~g})$. The surface tension ranged between $0.012 \mathrm{~N} / \mathrm{m} \leqslant \sigma \leqslant 0.0737 \mathrm{~N} /$ $\mathrm{m}$, the liquid density between $790 \mathrm{~kg} / \mathrm{m}^{3} \leqslant \rho \leqslant 1670 \mathrm{~kg} / \mathrm{m}^{3}$ and the Bond Number between $0.00065 \leqslant B o \leqslant 0.71$. The correlation predicts roughly $90 \%$ of the data within $\pm 10 \%$ with a maximum difference of $16 \%$. The average percentage difference between the experimental and predicted departure volumes is $4.7 \%$ with a standard deviation of $4 \%$.

Roughly, values of $V^{\prime}$ in the region of 1000 and above correspond with $V_{\text {det }} \sim V_{T}$ where the bubbles are quite spherical and are significantly larger than the corresponding orifice size. Below this, the contact pressure becomes increasingly more important and the bubbles tend to elongate to the extent that the bubble size at departure is similar to that of the orifice. Albeit developed from water measurements, Eq. (19) can be considered a general expression for the prediction of the bubble departure volumes from submerged orifice. This correlation differs from the preponderance of the bubble departure theories and correlations which exist in the literature which typically predict the bubble departure diameter or equivalent departure diameter based on the departure volume. This later method may well be applicable for cases where

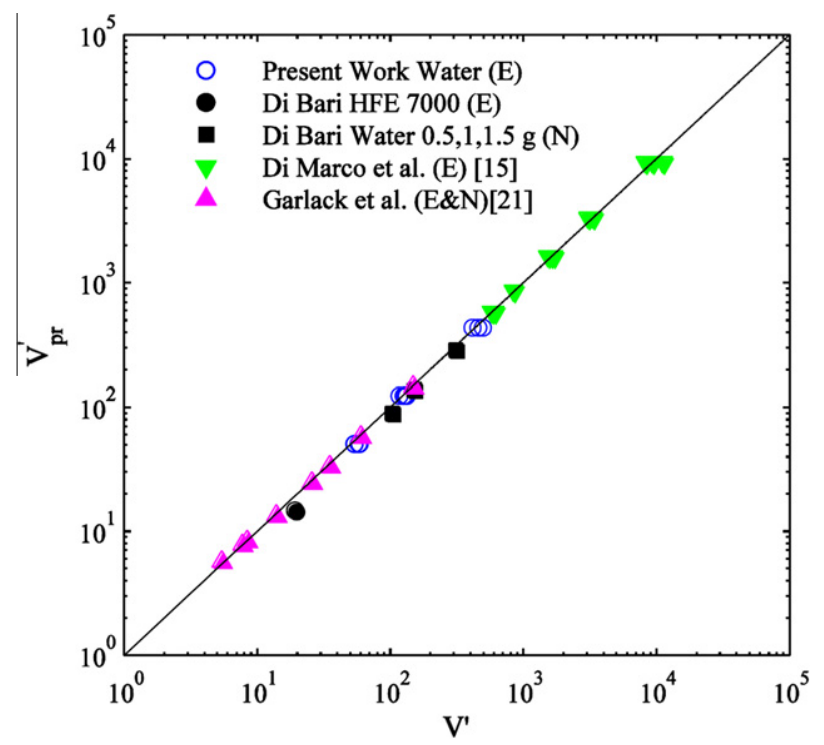

Fig. 18. Predicted versus measured and/or calculated dimensionless bubble departure volume: (E) experimental, $(\mathrm{N})$ numerical. 


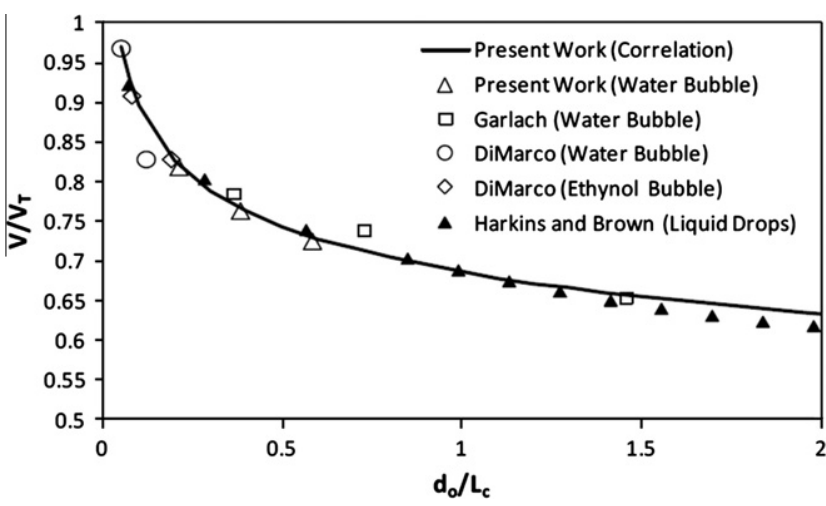

Fig. 19. Comparison of the predictions of Eq. (18) with drop weight data tabulated by Harkins and Brown [53].

$V^{\prime}>1000$ and the bubbles are quite spherical at departure, but becomes increasingly less appropriate for decreasing values of $V^{\prime}$ where the bubbles become progressively more elongated. Conversely, the proposed correlation does not require any assumption about the bubble shape at departure.

Harkins and Brown [53] proposed a similar functional relationship as that of Eq. (18) though for the volume of detached water droplets. Their investigation was focussed on developing an accurate drop weight technique for measuring the surface tension of liquids. They correctly noted that the law of Tate, i.e. that the weight of a drop is proportional to the tip radius and the surface tension $\left(m g=\pi d_{o} \sigma\right)$, was not strictly correct as it also depended on the shape of the drop. They hypothesised that this could be accounted for by introducing a dependence on a ratio of the tip radius with a linear dimension of the bubble, such as the capillary length, $L_{c}$. In this way, the weight of the liquid drop could be expressed as a relation such as $m g=\pi d_{o} \sigma f\left(d_{o} / L_{c}\right)$. In [53], the function $f\left(d_{o} / L_{c}\right)$ was termed a correction that should be used for translating drop weight data into surface tension measurements. It should be noted that Harkins and Brown [53] used the Capillary Number, a, to represent a linear dimension of the bubble related to its shape which is related to the Capillary Length used here through $a^{2}=2 L_{c}^{2}$. The ratio of the drop mass (or volume) to the Tate mass (or volume) can be expressed as,

$$
\frac{m g}{(m g)_{T}}=\frac{V}{V_{T}}=f\left(\frac{d_{o}}{L_{c}}\right)
$$

which was determined by weighing drops for different liquids and different tip radii. Fig. 19 shows a comparison between the predictions of Eq. (18) of this work with the drop weight data tabulated by Harkins and Brown [53] for their drop weight measurements which included data from previous researchers for low $d_{o} / L_{c}$. The data is only plotted for the approximate range of $d_{o} / L_{c}$ associated with the data in Fig. 18 (i.e. $0.05 \leqslant d_{o} / L_{c} \leqslant 1.7$ ) and a representative set of bubble measurements are also included in the plot. Interestingly, the current correlation accurately predicts the liquid drop weight data, even though it was developed from a limited set of bubble departure volume measurements. It is noted, however, that the Harkins and Brown [53] drop weight data reaches a local minimum at $d_{o} / L_{c} \sim 3$ (not shown) after which $f\left(d_{o} / L_{c}\right)$ subsequently increases. As a result, caution should be used in the implementation of Eq. (18) beyond the parameter range for which it has been shown to be valid.

\section{Conclusions}

Quasi-static bubble growth in water has been investigated experimentally at terrestrial gravity for different gas injection rates and orifice sizes. Considering the bubble shape and resultant pressure field revealed three distinct growth regions. Early growth is characterised by a bubble retaining a truncated spheroid-type geometry until the bubble volume subject to buoyancy increases to the extent that the hydrostatic pressure tended to elongate the bubbles during the mid growth stage. Finally, when the partial buoyancy force is large enough, the final stage occurs where a neck forms at the bubble base which collapses causing bubble break-off. The quasi-static growth and departure characteristics showed little dependence on the growth rate though a notable dependence on the orifice size. In particular, the smaller orifices produced smaller and more spherical bubbles that were generally much larger in proportion to the orifice size. These influenced the bubble shape, the relative contribution of the contact pressure force and the departure characteristics. On this last point, an uncomplicated empirical correlation has been formulated to predict the bubble departure volume over a broad range of fluid and orifice size combinations. The correlation appears valid for quasi-static bubble growth for bubbles with a fixed triple contact line in the range of $0.0007 \leqslant B o \leqslant 0.7$. The correlation shows very good agreement with the pendant drop data of Harkins and Brown [53] over the same range of Bond numbers.

\section{Acknowledgment}

We gratefully acknowledge the support from Science Foundation Ireland (SFI) through Grant Number: RFP/ENMF 249.

\section{References}

[1] F.W. Helsby, K.R. Tuson, Behaviour of air bubbles in aqueous solutions, Research 8 (1955) 270.

[2] J. Davidson, B. Schuler, Bubble formation at an orifice in a viscous liquid, Transactions of the Institution of Chemical Engineers 38 (1960) 144-154.

[3] A. Kupferberg, G. Jameson, Bubble formation at a submerged orifice above a gas chamber of finite volume, Transactions of the Institution of Chemical Engineers 47 (1969) 241-250.

[4] S. Ramakrishnan, R. Kumar, N. Kuloor, Studies in bubble formation - (i) bubble formation under constant flow conditions, Chemical Engineering Science 24 (1969) 731-747.

[5] S. Ramakrishnan, R. Kumar, N. Kuloor, Studies in bubble formation - (ii) bubble formation under constant pressure conditions, Chemical Engineering Science 24 (1969) 749-761.

[6] D. McCann, R. Prince, Regimes of bubbling at a submerged orifice, Chemical Engineering Science 26 (1971) 1505-1512.

[7] Y. Park, A. Tyler, N. de Nevers, The chamber orifice interaction in the formation of bubbles, Chemical Engineering Science 32 (1977) 907-916.

[8] J. Lin, S. Banerji, H. Yasuda, Role of interfacial tension in the formation and the detachment of air bubbles. 1. A single hole on a horizontal plane immersed in water, Langmuir 10 (1994) 936-942.

[9] N. Kyriakides, E. Kastrinakis, S. Nychas, A. Goulas, Bubbling from nozzles submerged in water: transitions between bubbling regimes, Canadian Journal of Chemical Engineering 75 (1997) 684-691.

[10] L. Zhang, M. Shoji, Aperiodic bubble formation from a submerged orifice, Chemical Engineering Science 56 (2001) 5371-5381.

[11] M. Jamialahmadi, M. Zehtaban, H. Muller-Steinhagen, A. Sarrafi, J. Smith, Study of bubble formation under constant flow conditions, Chemical Engineering Research and Design 79 (2001) 523-532.

[12] S. Gnyloskurenko, A. Byakova, O. Raychenko, T. Nakamura, Influence of wetting conditions on bubble formation at orifice in an inviscid liquid, Transformation of Bubble Shape, Colloids and Surfaces A: Physicochemical and Engineering Aspects 218 (2003) 73-87.

[13] A. Byakova, S. Gnyloskurenko, T. Nakamura, O. Raychenko, Influence of wetting conditions on bubble formation at orifice in an inviscid liquid: mechanism of bubble evolution, Colloids and Surfaces A: Physicochemical and Engineering Aspects 229 (2003) 19-32.

[14] H. Tsuge, Y. Tezuka, M. Mitsudani, Bubble formation mechanism from downward nozzle - effect of nozzle shape and operating parameters, Chemical Engineering Science 61 (2006) 3290-3298.

[15] G. Duhar, C. Colin, Dynamics of bubble growth and detachment in a viscous shear flow, Physics of Fluids 18 (2006) 077101.

[16] V. Buwa, F. Durst, Experimental investigations of regimes of bubble formation on submerged orifices under constant flow condition, Canadian Journal of Chemical Engineering 85 (2007) 257-267.

[17] H.N. Oguz, A. Prosperetti, Dynamics of bubble growth and detachment from a needle, Journal of Fluid Mechanics 257 (1993) 111-145. 
[18] D. Wong, C. Rumschitzki, C. Maldarelli, Theory and experiment on the low Reynolds number expansion, contraction and detachment of a bubble pinned at the tip of a submerged capillary, Journal of Fluid Mechanics 356 (1998) 93124.

[19] F. Higuera, Injection and coalescence of bubbles in a very viscous liquid, Journal of Fluid Mechanics 530 (2005) 369-378.

[20] Z. Xiao, R. Tan, An improved model for bubble formation using the boundaryintegral method, Chemical Engineering Science 60 (2005) 179-186.

[21] F. Higuera, A. Medina, Injection and coalescence of bubbles in a quiescent inviscid liquid, European Journal of Mechanics - B/Fluids 25 (2006) 164-171.

[22] Z.L. Yang, T.N. Dinh, R.R. Nourgaliev, B.R. Sehgal, Numerical investigation of bubble growth and detachment by the Lattice-Boltzmann method, International Journal of Heat and Mass Transfer 44 (2001) 195-206.

[23] B.K. Mori, W.D. Baines, Bubble departure from cavities, International Journal of Heat and Mass Transfer 44 (2001) 771-783.

[24] D. Gerlach, G. Biswas, F. Durst, V. Kolobaric, Quasi-static bubble formation on submerged orifices, International Journal of Heat and Mass Transfer 48 (2005) 425-438.

[25] S. Vafaei, P. Angeli, D. Wen, Bubble growth rate from stainless steel substrate and needle nozzles, Colloids and Surfaces A: Physiochemical and Engineering Aspects 384 (2011) 240-247.

[26] S. Quan, J. Hua, Numerical studies of bubble necking in viscous liquids, Physical Review E 77 (2008) 066303.

[27] A.K. Das, P.K. Das, Bubble evolution through submerged orifice using smoothed particle hydrodynamics: basic formulation and model validation, Chemical Engineering Science 64 (2009) 2281-2290.

[28] M. Ohta, D. Kikuchi, Y. Yoshida, M. Sussman, Direct numerical simulation of the slow formation process of single bubbles in a viscous liquid, Journal of Chemical Engineering of Japan 40 (2007) 939-943.

[29] V. Buwa, D. Gerlach, F. Durst, E. Schlucker, Numerical simulations of bubble formation on submerged orifices: period-1 and period-2 bubbling regimes, Chemical Engineering Science 62 (2007) 7119-7132.

[30] D. Gerlach, N. Alleborn, V. Buwa, F. Durst, Numerical simulation of periodic bubble formation at a submerged orifice with constant gas flow rate, Chemical Engineering Science 62 (2007) 2109-2125.

[31] Chakrabory, B. Ray, G. Biswas, F. Durst, A. Sharma, P. Ghoshdastidar, Computational investigation on bubble detachment from submerged orifice in quiescent liquid under normal and reduced gravity, Physics of Fluids 21 (2009) 062103.

[32] M. Ohta, D. Kikuchi, Y. Yoshida, M. Sussman, Robust numerical analysis of the dynamic bubble formation process in a viscous liquid, International Journal of Multiphase Flow 37 (2011) 1059-1071.

[33] I. Chakraborty, G. Biswas, P.S. Ghoshdastidar, Bubble generation in quiescent and co-flowing liquids, International Journal of Heat and Mass Transfer 54 (2011) 4673-4688.

[34] Y. Chen, M. Groll, Dynamics and shape of bubbles on heating surfaces: a simulation study, International Journal of Heat and Mass Transfer 49 (2006) 1115-1128.

[35] Y. Fujita, Q. Bai, Numerical simulation of the growth for an isolated bubble in nucleate boiling, in: Proc. of 11th International Heat Transfer Conference, vol. 2, 1998, pp. 437-442.
[36] G. Son, V.K. Dhir, N. Ramanujapu, Dynamics and heat transfer associated with a single bubble during nucleate boiling on a horizontal surface, Journal of Heat Transfer 121 (1999) 623-631.

[37] A.J. Robinson, R.L. Judd, Bubble growth in a uniform and spatially distributed temperature field, International Journal of Heat and Mass Transfer 44 (2001) 2699-2710.

[38] T. Fuchs, J. Kern, P. Stephan, A transient nucleate boiling model including microscale effects and wall heat transfer, Journal of Heat Transfer 128 (2006) 1257-1265.

[39] J.F. Klausner, R. Mei, D.M. Bernhard, L.Z. Zeng, Vapour bubble departure in forced convection boiling, International Journal of Heat and Mass Transfer 36 (1993) 651-662.

[40] T.H. Cochran, J.K. Aydelott, The effect of subcooling and gravity level on boiling in the discrete bubble regime, NASA Technical Note TN D-3449, 1966.

[41] Y.A. Buyevich, B.W. Webbon, Bubble formation at a submerged orifice in reduced gravity, Chemical Engineering Science 51 (1996) 48434857.

[42] L.Z. Zeng, J.F. Klausner, R. Mei R, A unified model for the prediction of bubble detachment diameters in boiling systems - I. Pool boiling, International Journal of Heat and Mass Transfer 36 (1993) 2261-2270.

[43] W.G.J. Van Helden, C.W.M. Van Der Geld, P.G.M. Boot, Forces on bubbles growing and detaching in flow along a vertical wall, International Journal of Heat and Mass Transfer 38 (1995) 2075-2088.

[44] P. Di Marco, Bubble growth and detachment: current status and future prospects, in: HEAT 2008, Fifth International Conference on Transport Phenomena in Multiphase Systems, Bialystok, Poland, June 30-July 3, 2008.

[45] E.S. Gaddis, A. Vogelpohl, Bubble formation in quiescent liquids under constant flow conditions, Chemical Engineering Science 41 (1) (1986) 97-105.

[46] P. Di Marco, N. Forgione, W. Grassi, Quasi-static formation and detachment of gas bubbles at submerged orifice: experiments, theorical prediction and numerical calculations, in: XXIII Congresso Nazionale UIT sulla trasmissione del calore, 2005.

[47] P. Di Marco, Birth, life and death of gas bubbles rising in a stagnant liquid, in: XXIII Congresso Nazionale UIT sulla trasmissione del calore, 2005.

[48] F. Bashforth, J.C. Adams, An Attempt to Test the Theory of Capillary Action, Cambridge University Press, 1892.

[49] S. Vafaei, D. Wen, Bubble formation on a submerged orifice, Journal of Colloid and Interface Science 343 (2010) 291-297.

[50] S. Siedel, S. Cioulachtjian, J. Bonjour, Experimental analysis of bubble growth, departure and interactions during pool boiling on artificial nucleation sites, Experimental Thermal and Fluid Science 32 (2008) 1504-1511.

[51] H. Wong, D. Rumschitzki, C. Maldarelli, Theory and experiment on the lowReynolds-number expansion and contraction of a bubble pinned at a submerged tube tip, Journal of Fluid mechanics (1998) 93-124.

[52] T. Tate, On the magnitude of a drop of liquid formed under different circumstances, Philosophical Magazine 27 (1864) 176-185.

[53] W.D. Harkins, F.E. Brown, The determination of surface tension (free surface energy), and the weight of falling drops: the surface tension of water and benzene by the capillary height method, Journal of the American Chemical Society 41 (1919) 499-524. 